\title{
Improving Delay-Based Data Dissemination Protocol in VANETs with Network Coding
}

\author{
Farhan H. Mirani ${ }^{1}$, Anthony Busson ${ }^{2}$, Cedric Adjih ${ }^{3}$ \\ 1 Signals and Systems Laboratory, Supelec, France \\ ${ }^{2}$ Laboratory of Parallel Computing, University of Lyon 1 - ENS - Inria, France \\ ${ }^{3}$ Hipercom Project-Team, Inria, France
}

Correspondence: Anthony Busson, anthony.busson@inria.fr

Manuscript communication: received 1 February 2013, accepted 25 March 2013

\begin{abstract}
In vehicular ad hoc networks (VANETs), for a large number of applications, the destination of relevant information such as alerts, is the whole set of vehicles located inside a given area. Therefore dissemination with efficient broadcast is an essential communication primitive. One of the families of broadcast protocols suitable for such networks, is the family of delay-based broadcast protocols, where farthest receivers retransmit first and where transmissions also act as implicit acknowledgements. For lossless networks, such protocols may approach the optimum efficiency. However with realistic loss models of VANET wireless communication, their performance is noticeably degraded. This is because packet losses have a double effect: directly on the amount of successfully received packets and indirectly with implicit acknowledgement misses. In this article, in order to combat the effects of packet losses, we combine delay-based broadcast with network coding, through a new protocol: Delay-based Opportunistic Network Coding protocol (DONC). By design, DONC aims at cancelling the twofold effects of packet and implicit acknowledgement losses. We describe the details of the DONC protocol, and we study its behavior, with realistic models and simulations. Results illustrate the excellent performance of the protocol.
\end{abstract}

Keywords- Wireless networks, Broadcast, Network Coding, VANET (Vehicular Ad hoc NETwork).

\section{INTRODUCTION}

In recent years, we have witnessed the emergence of a popular solution for future road communications: intervehicular communication (IVC). IVC systems have the potential to greatly influence the road security as well as to improve traffic flow by providing the drivers with critical route information such as upcoming obstacles and weather conditions. In addition to vital information related to road security, there exists a wealth of information sources and applications that will benefit the driver or passengers in mobile vehicles such as: traffic congestion reports, variable speed limits, ambulance accommodation, construction zone warnings, local or regional business locations, gas stations, web surfing, file sharing, etc.

Due to their highly agile nature, VANETs cannot work in mere client-server type configurations. Fortunately, the most promising applications in VANETs rely on broadcast communications: dissemination of messages warning about a danger, data dissemination from the embedded sensors (speed, inter-distance, etc.) for autonomous driving systems, advertisements, etc. The basic functioning of information dissemination by broadcasting is illustrated by the following scenario: on a route, a random mobile node encounters an event which needs to be notified to its neighbors. The mobile node that encountered the event will send information in the form of broadcast transmissions, which may subsequently be rebroadcasted by the receiving nodes to propagate the information to further distance from the source vehicle in multiple hops. This mechanism is called flooding. In a pure flooding mechanism, every receiving node will rebroadcast/relay the packet back to its neighbors. Obviously, such flooding is often inefficient because in many cases one node will rebroadcast information that was already received by all its neighbors, causing packet collisions and straining the already scarce radio bandwidth.

In our work, we follow the philosophy of a family of improved broadcasting schemes, denoted delay-based broadcasting schemes (according to the taxonomy of the survey [1]). The idea of delay-based broadcasting mechanisms is that among a group of receivers, the farthest node from the transmitter will retransmit a received packet, thus avoiding redundant simultaneous transmissions. This strategy approaches the optimum on linear topologies with lossless wireless transmissions, however, it does not perform so well in practical VANETs where wireless losses actually lessen the amount of self-elimination, and thus cause redundancy [2].

In order to give a precise overview of the context and the contributions of this paper, we detail in the two next paragraphs the principles of delay-based broadcasting schemes, useless transmissions arising with this scheme 
(e) Timer $=7 \mathrm{~ms}$

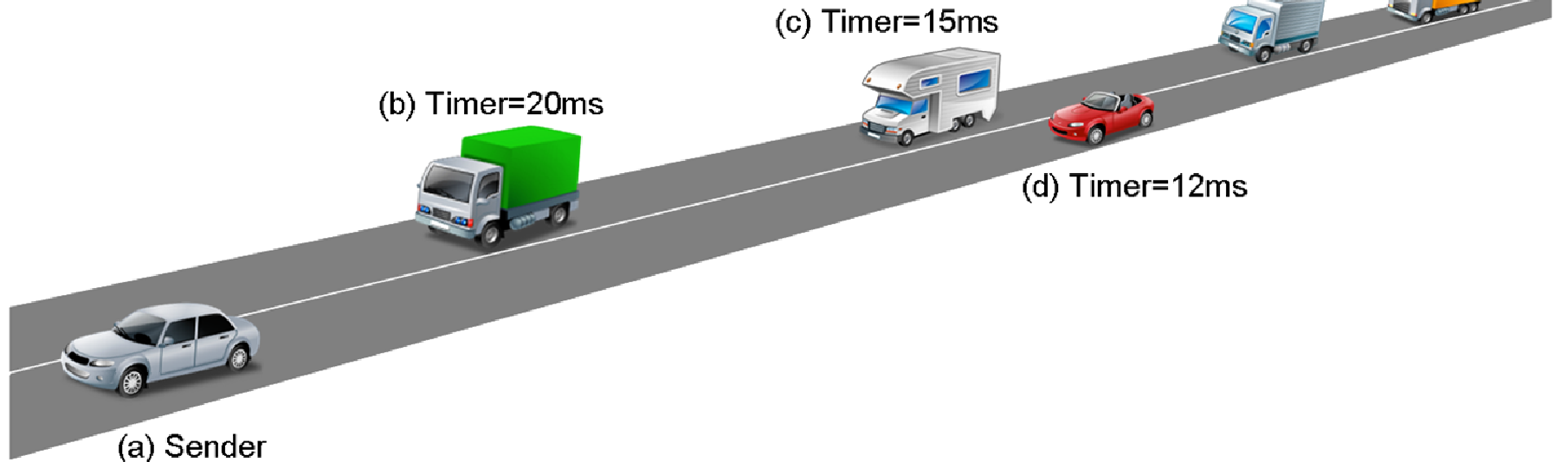

Figure 1. An example vehicular network

in lossy environment, and our contributions which consists in alleviating this problem through network coding techniques.

\subsection{Delay-based Broadcast Fundamentals}

In delay-based broadcasting, each node that receives a downstream packet does not rebroadcast it immediately. Instead, it starts a calculated timer governed by a 'delay' parameter. If by the end of the timer, the node does not receive the same packet from the opposite direction, only then it is allowed to rebroadcast. However, if the node receives the same packet as an upstream packet before its timer expires; it assumes that a vehicle further aheads received the same packet and broadcasted before it. Thus, this upstream packet is considered as an implicit acknowledgement at the receiving node and consequently the node cancels its currently running timer.

The value of the delay parameter, then, should not be pre-fixed for all the vehicles. Instead, the value of the delay parameter should take into account the current distance between the source and the receiving vehicles, such that the farther the receiver is from the source, the shorter the delay will be. Consequently, the farthest vehicle to have received a packet will have the shortest delay value of all, and therefore must be chosen as the relaying vehicle. Therefore,

$$
\text { Delay }=f(\text { distance }) \text {, }
$$

where $f$ is decreasing.

Figure 1 shows an example of a small vehicular network. From the example in Figure 1, vehicular node ' $a$ ' broadcasts a packet. Let us assume that the average broadcast coverage zone of node (a) covers the 4 vehicles behind. The broadcasted packet is received by nodes ' $b$ ', 'c', 'd', and 'e'. All four receiving nodes will then start a timer, where node ' $b$ ' will have a timer with the largest delay value $(20 \mathrm{~ms})$ and node ' $\mathrm{e}$ ' will have a timer with the smallest delay value $(7 \mathrm{~ms})$. Out of these four receiving nodes, the timer for node ' $\mathrm{e}$ ' will expire first and therefore node ' $\mathrm{e}$ ' will broadcast the packet.
Given the nodes ' $b$ ', ' $c$ ' and ' $d$ ' are in coverage range of node ' $\mathrm{e}$ ', they will receive this packet as well. Upon reception, these nodes will cancel their running timers, considering the received upstream packet as an implicit acknowledgement. This mechanism is repeated at each hop. The number of hops is set up by the application through a limited number of hops (the IP TTL field for instance), or information on the geographical coverage of the messages. This scheme is very efficient from the point of view of implementation/complexity as well as redundancy. It does not rely on any underlying protocol, such as routing, link management (Hellos), or any table. The only required information is the distance between the transmitter and the receiver. Geographical locations are easily obtainable through GPS, or any geographical systems. Therefore, the transmitter just needs to add its location in a message's field to allow a receiver to compute this distance. Moreover, this scheme systematically selects the best forwarder: the farthest one from the previous transmitter. It should lead to the minimization of the number of transmitters and thus redundancy.

In practice, it holds in an ideal radio environment, where the Frame Error Rate (FER) is 0 if the distance between the transmitter and the receiver is less than a given distance (the radio range), and 1 otherwise. This radio model is the so-called Boolean model. For such a model and the delay-based scheme, a node receives exactly two times each message: one time from the transmitter upstream and one time downstream. Unfortunately, it does not hold when the radio model is more complex, i.e. when the radio range is not a perfect ball. In Figure 2, we plotted the mean number of receptions for a disseminated message with regard to the traffic density (number of vehicles per kilometer). We do not present simulations' parameters here, as this curve is just used to highlight effects of the radio environment on the performances. Simulations parameters and results will be presented in detail in Section 4.3. We considered three different radio models, the Boolean, and two more realistic radio models: $2 \mathrm{RM}$ and Rayleigh. We observe that for the Boolean model, the delay-based 
scheme works perfectly, and the mean number of receptions is exactly 2 . For the two lossy models, this number increases significantly with the traffic density. In the worst case, it reaches approximately 4.7 receptions in average. This useless redundancy is mainly due to the implicit acknowledgement mechanism. Let us consider the previous example depicted in Figure 1. Vehicles ' $b$ ', ' $c$ ', ' $d$ ' and ' $e$ ' receives the message. Node ' $e$ ' retransmits first as it has the smallest timer. But, if ' $\mathrm{e}$ ' has not the same radio range as ' $a$ ', or if the radio range is not a perfect ball, the broadcast from ' $\mathrm{e}$ ' may not be properly received by ' $b$ '. In this case, ' $b$ ' not receiving the implicit acknowledgement from a vehicle downstream (from ' $\mathrm{e}$ '), forwards the message.

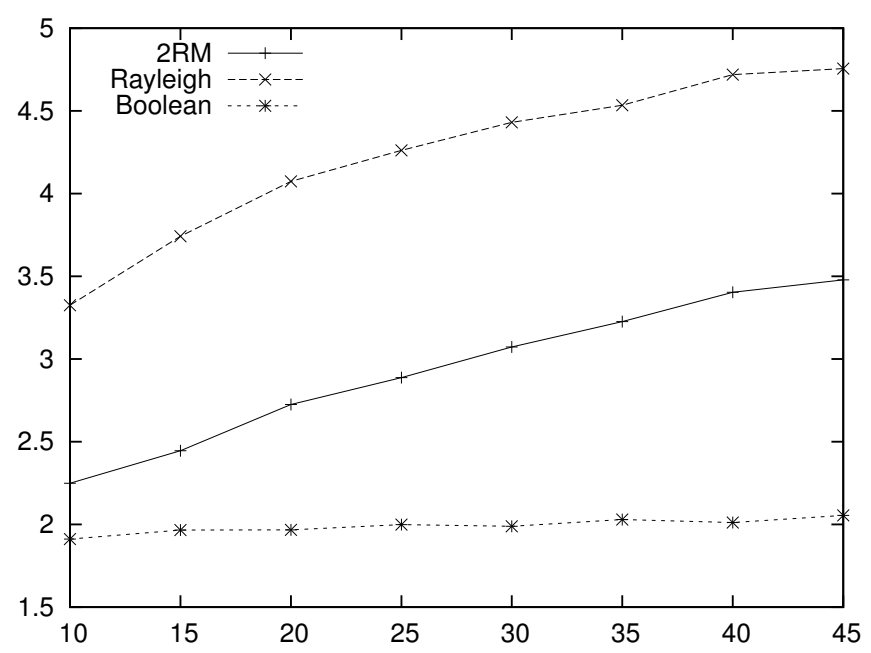

Figure 2. Mean number of receptions with a delay-based schemes for different radio models

\subsection{Contributions}

To combat this effect, we combine such a delaybased scheme with network coding [3]. Network coding is a recent transmission paradigm where intermediate nodes in the network can code different incoming packets instead of just forwarding them as-is. Network coding is known to yield capacity gains and to improve resilience to losses.

This paper presents DONC, a Delay-based Opportunistic Network Coding protocol. DONC exploits the broadcast nature of the wireless medium to implement a distributed network coding scheme specifically tailored for lossy VANETs. Instead of transmitting individual packets as received, DONC linearly encodes the original packets into coded packets before a (delaybased) retransmission. Decoding a coded packet becomes possible when enough decoding information is received at a node. Upon decoding, original packets are extracted and only then these packets are allowed to be further retransmitted. DONC features four important properties:

- Delay-based broadcast: this optimized flooding mechanism with implicit acknowledgements is the basis for DONC.
- Stop-and-Go: a node will retransmit coded packets only after it has been able to decode them. This yields more control on the flooding process.

- Resilience to losses: with network coding, different nodes may still decode coded packets subject to different loss patterns without requiring retransmissions on a per-packet basis (which is more expensive).

- Resilience to losses in overhearing: one retransmission will act as an implicit acknowledgment for several source packets instead of just one, increasing the probability of self-elimination in lossy settings, hence performance.

\subsection{Organization}

The rest of this paper is organized as follows: Section 2 discusses the general background of our work, including its context and fundamental ideas. In Section 3, we propose an efficient data dissemination protocol for lossy VANETs called DONC protocol. Performance evaluation of the proposed protocol is presented in section 4 and before concluding our paper in Section 6, Section 5 reviews some important works proposed on broadcast in vehicular networks as well as on network coding.

\section{General Background}

\subsection{Context and Assumptions}

Our context here is of a completely distributed vehicular network (preferably V2V), where each vehicle is a mobile node in a vehicular ad hoc network (ref. Figure 1). These vehicles are configured to transmit in broadcast mode and may transmit diverse information to other neighboring vehicles regarding road conditions, weather updates, accident warnings, traffic information, advertisements, etc. Due to high vehicle mobility, we assume the network conditions may vary from stable and predictable to highly lossy and unpredictable. A node may be able to communicate information with neighboring nodes efficiently for an extended period of time, or it may only get enough window of time to transmit a quick burst of data to convey a message to a node closest to it.

In a general VANET, a vehicle may receive either downstream or upstream packets: in the example in Figure 1, for node ' $c$ ', all the packets received from nodes ' $a$ ' and ' $b$ ' are upstream and all the packets from nodes ' $\mathrm{d}$ ' and ' $\mathrm{e}$ ' are downstream. In order to keep the context of our work simple at this point, we consider that the propagation of data is carried out in only one direction, in the upstream direction (from right to left in Figure 1, and with downstream packets): this direction is the most relevant for typical VANET applications, since it can convey information on the environment that will be met later by the vehicles (places of interest, incidents,...).

Finally, we assume all the vehicular nodes involved are equipped with GPS receivers so that each receiving 
vehicle can deduce its distance from the transmitting vehicle.

\subsection{Network Coding Operation}

We assume every vehicle in the network can not only send and receive packets of data but can also perform network coding operations. Network coding allows a node to "mix" two or more data messages into a coded packet and broadcast it. This mixing of data messages is referred as 'packet encoding'. Upon reception of a data packet, the receiving vehicle tries to 'decode' it with the information it currently possesses. For the sake of simplicity, we assume a data packet to be a container of one or more unique data messages. If a data packet contains only one message, it is considered not-coded and hence its contents can be retrieved on its own, without any further information. However if a packet contains more than one messages in it, the data packet is considered to be encoded and it can only be decoded if sufficient decoding information is present at the receiving node.

For detailed information about network coding operations in our work, readers are referred to related work (section 5) and cited works: MORE [4] and OMNC [5], which explain in detail network coding models identical to ours. In summary: packets are supposed to be of identical size (concatenated and padded as necessary), coded packets are generated from source packets with linear combinations through random linear coding [6], decoding is performed with Gaussian elimination and encoding vectors are inserted in headers [7] (with a mapping from packet indices to node and packet identifiers; finding efficient mappings is not addressed by this article, see [8] for instance).

\section{DONC PRotocol}

DONC, or Delay-based Opportunistic Network Coding protocol fuses the idea of the popular delay-based broadcasting mechanism for VANETs with the principles of network coding, which allow to improve network throughput and reduce packet loss in error and collision prone wireless networks.In order to help readers understand the basic idea of our protocol without entering immediately in the algorithm and implementation details, we split this section in two parts. We first present the basic idea and functioning of DONC protocol. Also, we present a simple example that highlights the benefit of DONC with regard to the basic delay-based scheme. Then, we present the protocol details. In order to implement network coding with a delay-based broadcasting mechanism, we propose to introduce an intermediate layer in the protocol stack called the Network Coding (NC) layer, as shown in the figure 3. In the NC layer, we introduce two distinct buffers to store incoming data packets: the Data-Buffer and the Decoding-Buffer.

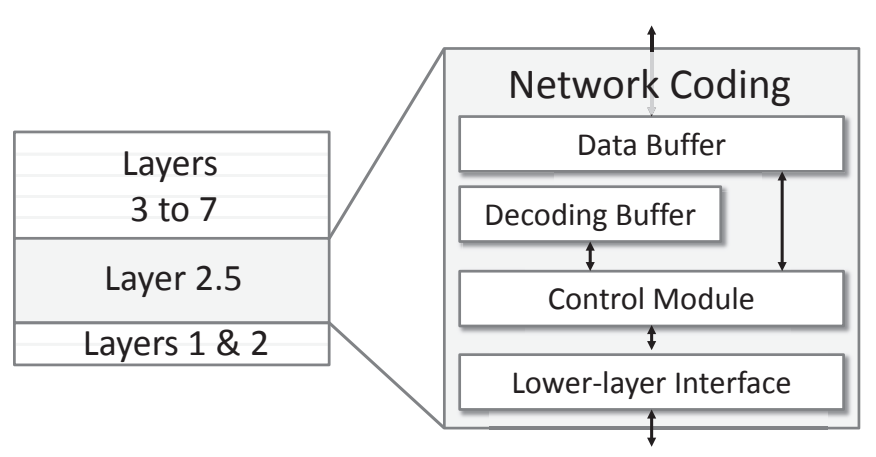

Figure 3. Network Coding (NC) Layer - Decomposition

\subsection{Protocol Semantics and Architecture}

3.1.1 Overview: The objective of the protocol is the broadcast of data packets. They are stored in two data structures (described later):

- Data-Buffer: It is a buffer that stores decoded/notcoded data packets.

- Decoding-Buffer: It is a temporary buffer to store incoming coded data packets which cannot immediately be decoded at the receiving vehicle with Gaussian elimination and require additional coded data packets.

The NC layer also has a Control Module and a Lower Layer Interface module. The Control Module is responsible for all the operations of the protocol, which include fetching data to and from the Databuffer and Decoding-buffer, running packet decoding procedures, keeping track of timers, etc. Lower Layer Interface module provides interface between NC layer and layer- 2 .

The functioning of DONC is based on the following principles:

- Coded packets stay in the decoding buffer until decoded,

- Reception from upstream of a given packet acts as an implicit acknowledgement and any scheduled (re)transmission of the packet is canceled,

- Decoded/non-coded packets are transmitted after an initial delay-based timer (unless canceled),

- Decoded/non-coded packets are later retransmitted until implicit acknowledgement,

- Any transmission of decoded/non-coded packets is performed by first computing a linear combination of such available packets (network coding).

3.1.2 Semantics: DONC semantics rely on three kinds of events: local application packet handling, packet reception and coded packet transmission.

\section{Local Application Packet Handling}

- Every data packet locally generated by the application(s) of a node is added to the Data-Buffer with its transmission timer set to current time; hence a packet transmission is immediately performed.

\section{Packet Reception}

- Upon receiving a coded packet, it is directly added to the Decoding Buffer. Thereafter, a decoding of the current set of the coded packets with Gaussian 
elimination is attempted. This may result in the decoding of zero, one, several or all the coded packets in the Decoding Buffer.

- Decoded packets are then removed from the Decoding Buffer, and for each packet decoded:

- if it is not already present in the data buffer, it is added

- otherwise, if it is an upstream packet, it is considered to be an implicit acknowledgment and any associated retransmission timer is removed.

- otherwise it is ignored and dropped

\section{Coded Packet Transmission}

- Whenever the transmission timer of an uncoded packet expires:

- the first $N$ uncoded packets with the lowest expiry timers are coded together and transmitted, where ' $\mathrm{N}$ ' be the maximum number of messages allowed to be encoded in a data packet

- their timers are updated with the retransmission timeout

3.1.3 Data Structures: Every node in the network has a reference table (Ref-Table), which keeps information regarding packet transmissions, timers and acknowledgements. It has three fields: Msg-Ids, Ack-Flgs and Exp-Timers corresponding to each data message in the Data-buffer (ref. table I). These entries are updated every time a vehicle has some activity (transmission or reception). Since Ref-Table is a reflection of the current contents of Data-Buffer, the size of Ref-Table is equivalent to that of the Data-Buffer.

- Msg-Id: It is a unique ID given to each message before it is encoded in a data packet. A data packet may contain many messages and hence many MsgIds. Each vehicle uses a unique tag (originator IP address and packet sequence number) to differentiate from the packets originating at neighboring nodes.

- Ack-Flg: It is a flag which indicates if a particular message has been acknowledged or not.

- Exp-Timer: Every message in the data buffer is associated an expiry time, after which the message should be (re)broadcasted. Exp-Timer field indicates the time when the vehicle wants this message to be (re)broadcasted.

Table I

Example of a ReF-Table

\begin{tabular}{|c|c|c|}
\hline Msg-Id & Ack-Flg & Exp-Timer \\
\hline 01 & 1 & $1.021554 \mathrm{sec}$ \\
\hline 02 & 0 & $1.121245 \mathrm{sec}$ \\
\hline 03 & 0 & $1.245644 \mathrm{sec}$ \\
\hline
\end{tabular}

\section{Implicit Acknowledgments: Ack-Flg Update}

Our protocol operates with implicit acknowledgements exclusively. When a vehicle receives a second copy of a particular packet from one of its neighboring vehicles, it checks if it is a downstream or upstream packet. In case it is a downstream packet, it is considered to be an unnecessary retransmission and hence is discarded upon reception. However if it is an upstream packet, it means the transmitting vehicle has just forwarded this packet further ahead, so the node considers this as an implicit acknowledgement. The value ' 0 ' in the Ack-Flg field indicates that an acknowledgement is still awaited for the corresponding message. ' 1 ' means the message is already acknowledged and accounted for.

\section{Timer: Exp-Timer Update}

The initial value to be given to Exp-Timer is calculated as:

$$
\text { Exp }- \text { Timer }=t+\text { Delay, }
$$

where ' $t$ ' is the current system time and Delay is a decreasing function of the distance between the transmitter and the receiver. Delay is ' 0 ' if the message is generated by the broadcasting vehicle itself, which means this message needs to be transmitted immediately. After the first expiry of the timer, the timer becomes a timeout ensuring retransmission until an implicit acknowledgement is received (or a limit is reached) as detailed in section 3.2.3.

3.1.4 Example: In Figure 4, we illustrate an example of delay-based scheme with and without network coding. There are 10 vehicles, labelled from ' $a$ ' to ' $j$ '. We assume that packets must be disseminated in one direction (it is generally upstream with regard to the vehicles' traffic), from node ' $a$ ' to ' $j$ '. Nodes ' $a$ ' and 'd' generate a packet, respectively $P_{1}$ and $P_{2}$. Arrows represent radio ranges of the transmissions. Numbers within the nodes indicate the number of receptions after each step. For the delay-based scheme without network coding (Figure 4(a)):

Step 1 nodes ' $a$ ' and ' $d$ ' transmit the two packets $P_{1}$ and $P_{2}$,

Step 2 node ' $\mathrm{f}$ ' being the farthest receiver from ' $a$ ', it retransmits the packet $P_{1}$. Node ' $h$ ' does the same for $P_{2}$.

Step 3 But, node ' $b$ ' does not receive properly the retransmission from ' $f$ '. As it has not received any implicit acknowledgement, node ' $b$ ' retransmits $P_{1}$ at the expiration of its timer. At the same time, node ' $\mathrm{j}$ ' retransmits $P_{1}$ as it is the farthest receiver from ' $\mathrm{f}$ '.

We consider exactly the same scenario for the delaybased scheme using network coding (Figure 4(b)):

Step 1 when node ' $d$ ' waits for its timer associated to $P_{1}$, it receives a packet $P_{2}$ from a local application. Then, it combines these two packets using network coding leading to only one packet $P_{1}+{ }_{1} P_{2}{ }^{1}$, and transmits it immediately. It does not wait for the timer expiration since it has a local packet to disseminate.

Step 2 Nodes ' $\mathrm{b}$ ', ' $\mathrm{c}$ ', ' $\mathrm{e}$ ' and ' $\mathrm{f}$ ' have received $P_{1}$ and $P_{1}+{ }_{1} P_{2}$ and are thus able to decode $P_{2}$. The packet $P_{1}+{ }_{1} P_{2}$ is used as an implicit acknowledgement for both packets $P_{1}$ and $P_{2}$. All the nodes downstream ' $d$ ' (nodes ' $a$ ', ' $b$ '

\footnotetext{
${ }^{1}$ We use $+_{1},+_{2}$, etc. to indicate that the coding operations/coefficients are different for each new combination.
} 


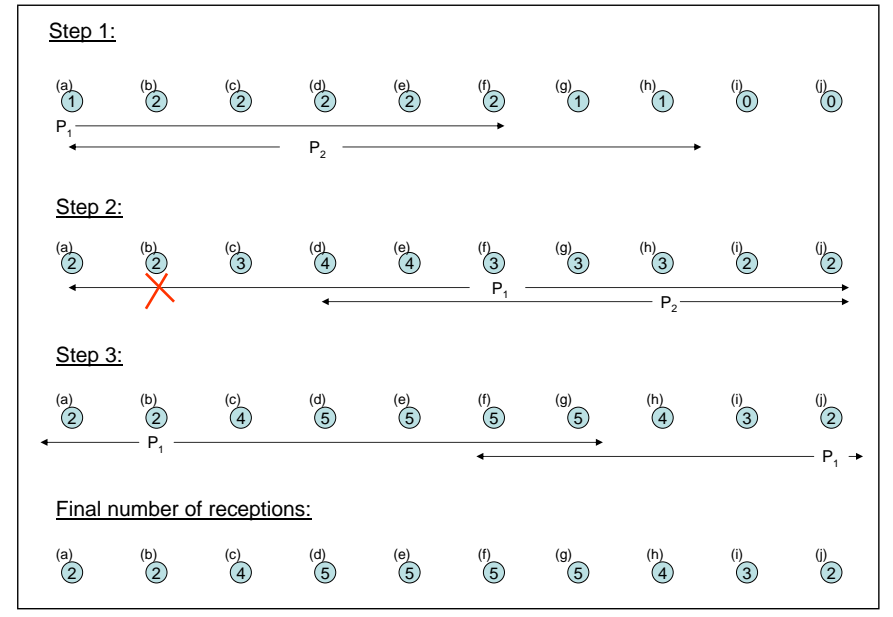

(a) Delay-based scheme without network coding

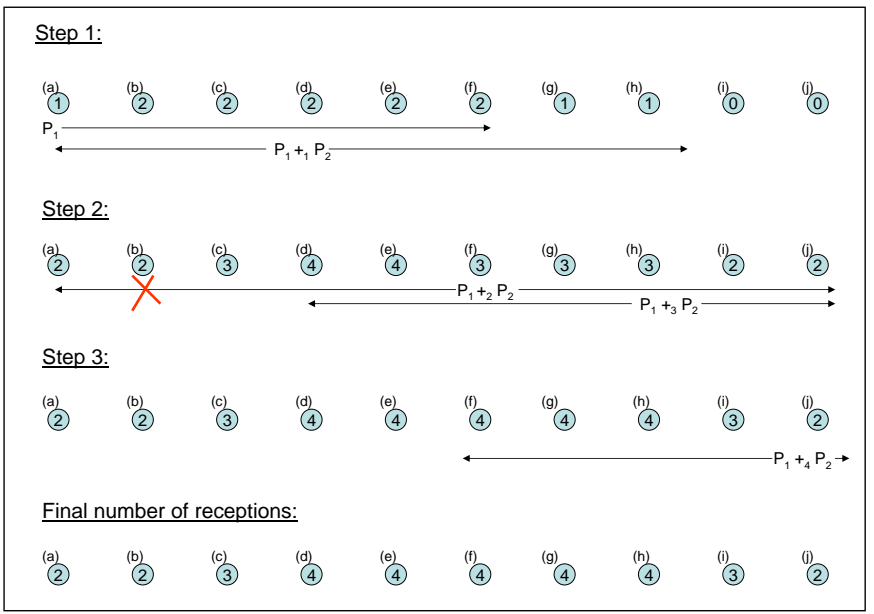

(b) Delay-based scheme with network coding

Figure 4. An example of the dissemination of two messages with and without network coding. Node 'a' generates a packet $P_{1}$ and node ' $\mathrm{d}$ ' a packet $P_{2}$. The retransmission from ' $\mathrm{f}$ ' is not received by ' $\mathrm{b}$ '.

and ' $c$ ') cancel timers and retransmissions for these two packets. Nodes ' $g$ ' and ' $h$ ' have received only $P_{1}+{ }_{1} P_{2}$. This packet stays in the decoding buffer waiting to be decoded. Nodes ' $\mathrm{e}$ ' and ' $\mathrm{f}$ ' have two packets in their data buffer (decoded packets). According to our protocol, there are two timers associated to these two packets. The first timer that expires is the timer at node ' $\mathrm{f}$ ', because it is farther from node ' $a$ ' and thus a shorter delay. Therefore, node ' $\mathrm{f}$ ' combines all the packets in its data buffer $\left(P_{1}\right.$ and $\left.P_{2}\right)$. It generates a new coded packet $P_{1}+{ }_{2} P_{2}$ and transmits it.

Step 3 Nodes ' $\mathrm{g}$ ' and ' $\mathrm{h}$ ' are now able to decode $P_{1}$ and $P_{2}$. Node ' $h$ ' being the farthest receiver that have decoded packets, it is the next forwarder.

We observe that without networking coding, it suffices that a node does not receive an implicit acknowledgement to generate a useless transmissions. Such an event, occurring with a high probability as the vehicles' density is great, it may increase significantly the number of useless receptions and reduce the available bandwidth. The benefit of our approach lies in the fact that the coded packets acknowledge several packets. Consequently, it admits a certain number of losses without degrading the performances. A detailed evaluation of DONC will be presented in Section 4.3.

\subsection{DONC Protocol Detailed Functioning}

In this section, the functioning of the DONC Protocol is detailed in response to the three events listed in the previous section. The functioning is represented by flow-diagrams in Figure 5, Figure 6 and Figure 7.

3.2.1 Detailed Local Application Packet Handling: The $\mathrm{NC}$ layer is responsible for the execution of DONC protocol, whose behavior depends upon the side the NC layer receives packets from; either an 'outgoing' or an 'incoming' data packet. An outgoing packet is a message generated by a local application running on the transmitting vehicle, and hence the Network
Coding (NC) layer receives it from the higher layers. On the other hand, an incoming packet is the one received by a vehicle from one of its neighbors. It may contain a single message or a combination of several messages in it. The NC layer receives it from the lower layers and delivers it to the higher layers after having processed it. Every time there is some information to be broadcasted

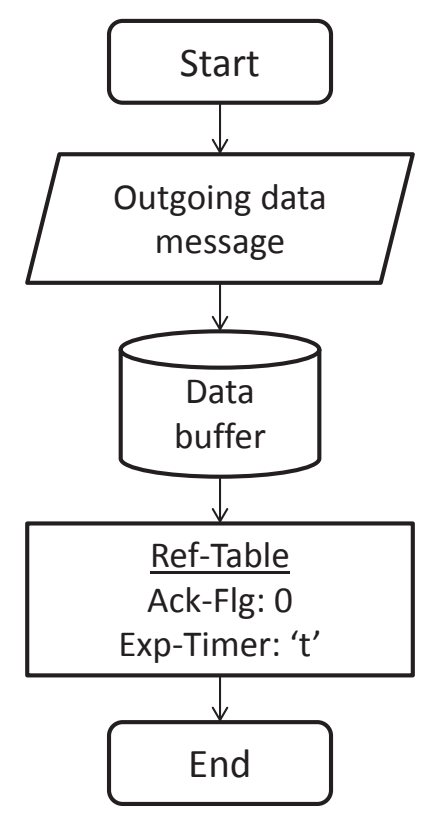

Figure 5. NC Layer - Local Application Packet Handling

from a local application, the NC layer receives it from higher layers in the form of an outgoing packet. The network Coding layer first sends it to be stored in the Data-buffer. The NC layer then creates an entry in the reference table corresponding to the newly received message with its message ID number, flag set to ' 0 ' and expiry timer set to current system time. Finally, the NC layer starts the packet transmission routine. Flowdiagram of this process can be seen in figure 5 .

3.2.2 Detailed Processing for Packet Reception: Similarly, a flow-diagram for incoming packets is shown in fig- 
ure 6. Every time a vehicle receives a packet from a neighboring vehicle, the NC layer receives it from the lower layers. The NC layer first checks if the packet can be decoded with the information currently available at the vehicle (Data-buffer and Decoding-buffer). If it cannot be decoded, the NC layer sends the coded packet to be stored temporarily in the Decoding-buffer and wait for more information to be received. On the other hand if the received packet can be decoded, the NC layer extracts individual data messages from it. Upon

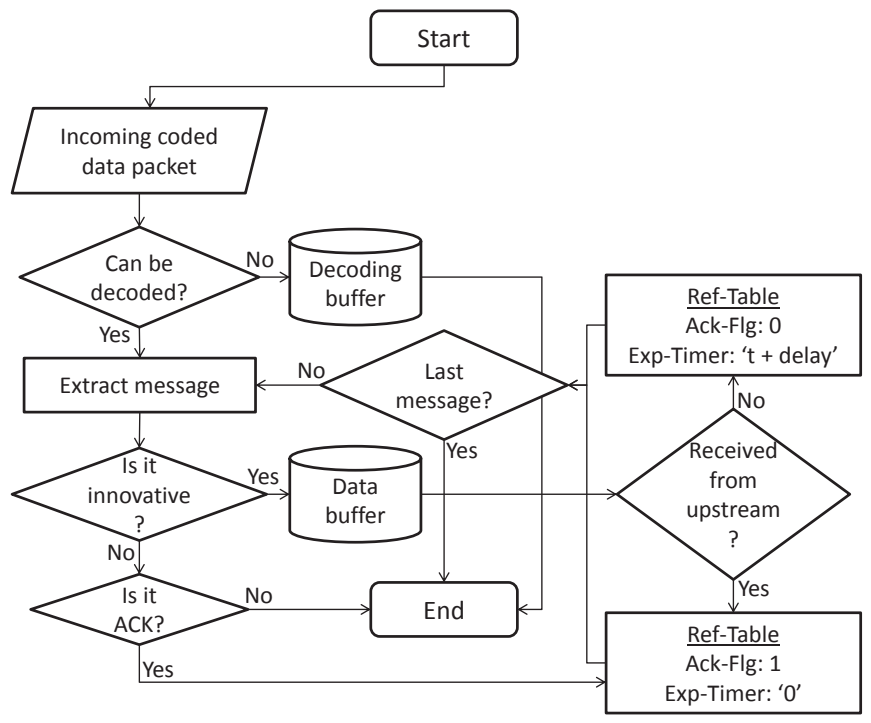

Figure 6. NC Layer - Packet Reception

extraction, it is first checked whether each message is innovative or not. The innovative messages are sent to be stored in the Data-buffer and their respective reference table entries are created (Msg-Id, Ack-Flg and Exp-Timer). Every time an innovative message is added to the Data-buffer, the NC layer checks if there are any coded packets in the Decoding-buffer that can now be decoded with the help of this newly received innovative message. If a coded packet is decoded, its contents are sent to the Data-buffer and the coded packet itself is deleted from the Decoding-buffer. On the other hand, the newly decoded non-innovative messages may either be acknowledgements or unnecessary retransmissions. To verify which category of the two they fall in, the NC layer checks if the incoming packet was a downstream or upstream packet. A downstream packet will mean the message was a retransmission, and therefore, is immediately discarded without further inquiry. If however, the message was received in an upstream packet, it means this message is an acknowledgement of a previous broadcast. Therefore, NC layer will update the reference table entry corresponding to the received acknowledgement by flipping its flag from ' 0 ' to ' 1 '.

After every activity on the Data-buffer, the buffer is sorted in ascending order by the values of its Exp-Timer field, so that the elements with the smallest values are on the top. The NC layer then assigns the timer the value of the first element from the top of the reference table whose Ack-Flg is not ' 1 ', i.e., the corresponding message is not already acknowledged. From the exam- ple given in table I, although the Exp-Timer value of message ' 01 ' is smaller than that of message ' 02 ', but the vehicle timer skips the first value because the message is already acknowledged and takes the value of ExpTimer of message '02' instead.

3.2.3 Detailed Coded Packet Transmission: On a vehicle, NC layer starts the packet transmission routine (ref. flow-diagram in figure 7) as soon as its timer expires. First, the NC layer picks the first ' $\mathrm{N}$ ' messages from the reference table whose Ack-Flgs are ' 0 '. It then encodes these ' $\mathrm{N}$ ' messages in a coded data packet, before sending it to the lower layers for broadcast. NC layer then updates the Exp-Timer values in the reference table for the corresponding messages as:

$$
\text { Exp }- \text { Timer }=t+\text { Ret_Timeout, }
$$

where 'Ret_Timeout' is the retransmission timeout, a constant set to a value of the order of magnitude of the time necessary for a packet to be received and acknowledged by at least one of the neighboring vehicular nodes.

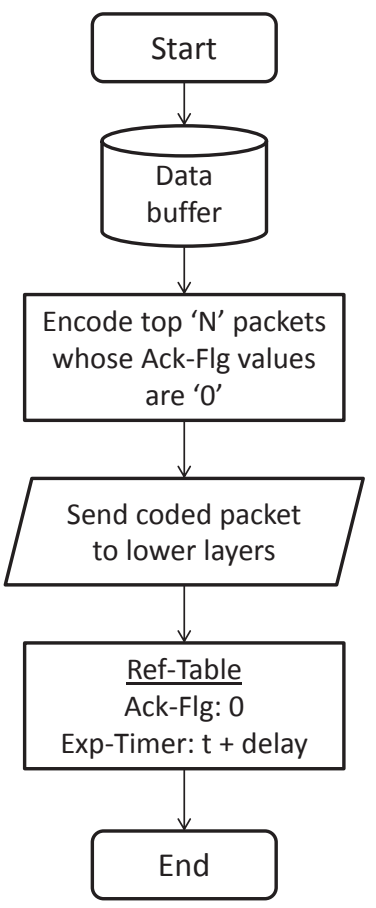

Figure 7. Coded Packet Transmission Routine

\section{Performance Evaluation}

In this section, we evaluate the performance of DONC protocol by simulation. We use Network Simulator 2 (ns2) [9], which is an open source discrete event network simulator. We compare the performance of DONC protocol with a standard delay-based broadcast mechanism. To avoid unnecessary repetition of words, we call it the SDB (Standard Delay-based Broadcast) protocol. The presented results illustrate how combining network coding with a simple delay-based VANET broadcasting mechanism may help improve its performance, specially in adverse network conditions. 


\subsection{Simulation Scenario}

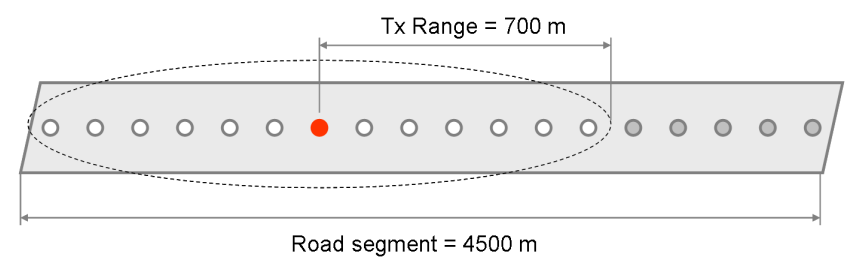

Figure 8. Simulation Topology

The topology we chose to test DONC protocol is as shown in figure 8. It consists of a fixed road segment of 4.5 kilometers approximately. All the vehicular nodes are equipped with radio equipment on the specifications of IEEE 802.11p standard. IEEE 802.11p is an enhancement to the IEEE 802.11 standard destined at adding Wireless Access in Vehicular Environments (WAVE) [10]. The vehicles in our topology are configured to transmit in a radius of 800 meters approximately, which corresponds to the 802.11p standard. Furthermore, in order to obtain results that are easier to interpret, we assume that the vehicles are regularly distributed (e.g identical inter-vehicle distance) and for each test, we vary the node density (from $5 \mathrm{veh} / \mathrm{km}$ to $45 \mathrm{veh} / \mathrm{km}$ ). For the sake of simplicity, we simulate a broadcast of 100 packets $\left(p k t_{1}, p k t_{2}, p k t_{3}, . ., p k t_{10}\right)$ from 10 first nodes $\left(n_{0}, n_{1}, . ., n_{10}\right)$, chosen randomly with a time interval of $1 \mathrm{~ms}$ between each broadcast. In order

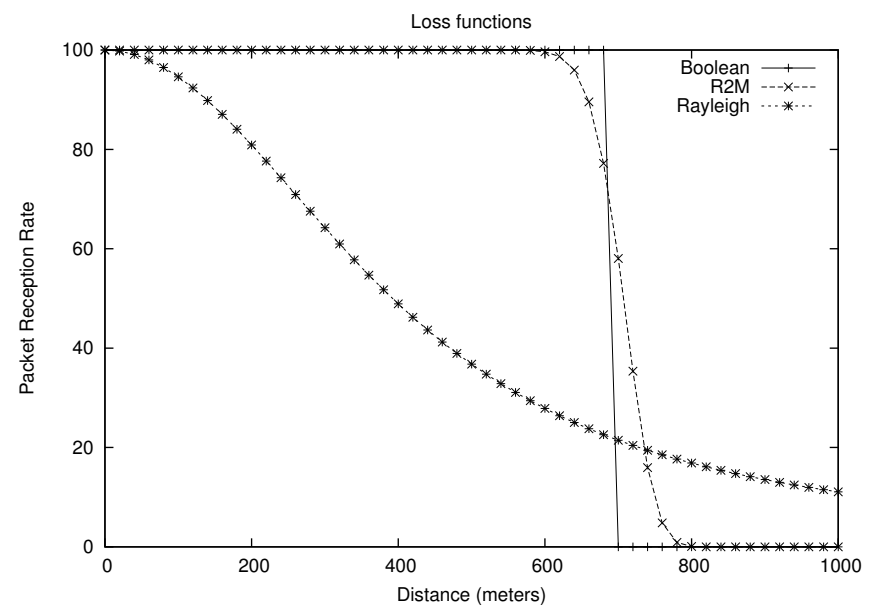

Figure 9. Boolean, 2RM and Rayleigh loss functions

to test DONC protocol in different wireless scenarios, we configured three distinct FER (Frame Error Rate) models (shown in figure 9), one for each ideal (no-loss), rural (scarce population) and urban (dense population) environments.

- The 2RM FER model was proposed in [11]. It is a measurement based model of the frame error process in rural setting. The model takes into account 802.11p wavelength, heights, distances, antenna gains, frame length, etc. Figure 9 presents the average packet reception rate for $2 \mathrm{RM}$ loss model.

- The Rayleigh FER model is destined for more complex, urban settings. In our case, the Rayleigh model serves as the 'worst case scenario' where FER changes frequently and does not present a definite threshold function. From the figure 9, it can be noted that packet reception rate for Rayleigh decreases quickly, even for small distances.

- The Boolean FER model is a custom-built ideal radio model designed to compare with the performances of more real-like radio models with little and heavy radio losses. The packet reception rate in this model is 1 for $x$ in $[0,700]$ and 0 for $x>700$.

For the delay selection in( 1$)$, the delay is a linear function of the distance with: Delay $=\beta-\alpha \times$ distance (for our simulations, $\beta=0.36$ and $\alpha=0.0005$ with distances expressed in meters and times in seconds).

\subsection{Illustration of Protocol Behavior with Sample Simulations}

The figure 10 illustrates a view of the transmissions and receptions, occurring in one sample simulation with a node density of $30 \mathrm{veh} / \mathrm{km}$.

It focuses on some transmissions occurring between time $0.7 \mathrm{sec}$ and time $1.0 \mathrm{sec}$ after the beginning of the simulations. We considered the whole set of transmissions occurring in the network; and after ordering them according to their occurrence time, we numbered them and selected the $40^{\text {th }}$ to the $80^{\text {th }}$ transmissions. This transmission numbering (index) is represented on the $y$-axis. On the $x$-axis, we represent the node identifier (which is directly proportional to the distance of the node from some reference point, because nodes are regularly spaced).

Then the diagram represents the impact of the $y^{\text {th }}$ transmission on the $x^{\text {th }}$ node:

- If the node is actually the emitter in the transmission, then depending whether the node sent an uncoded packet, or a linear combination of packets, respectively a yellow disk or a black square is represented.

- If the node is actually a receiver of the transmission, and if additionally it is further from the source, then a blue dot is represented.

Thus the diagram is an illustration of three aspects of the process of the optimized broadcast with DONC:

The first one is related to the Rayleigh model: we observe that each transmission is associated with a noncontinuous set of blue dots. Whereas, if there was no random loss (for instance with the boolean model), the set of blue dots would be continuous. Hence, the diagram gives an insight of the issues met by delaybased broadcast protocols without network coding: in order to ensure that all nodes receive one packet, they actually have to cover the $x$-axis with such disconnected (random) blue sets, for each transmission, which is difficult and costly.

The second one is related to the parameters selected for the simulations: about half of the packets appear not to be coded (for this whole simulation, statistics indicate that $48 \%$ of the packets are not coded). This is typical of other simulation scenarios presented in this article, and it shows the fact that even with "light" 


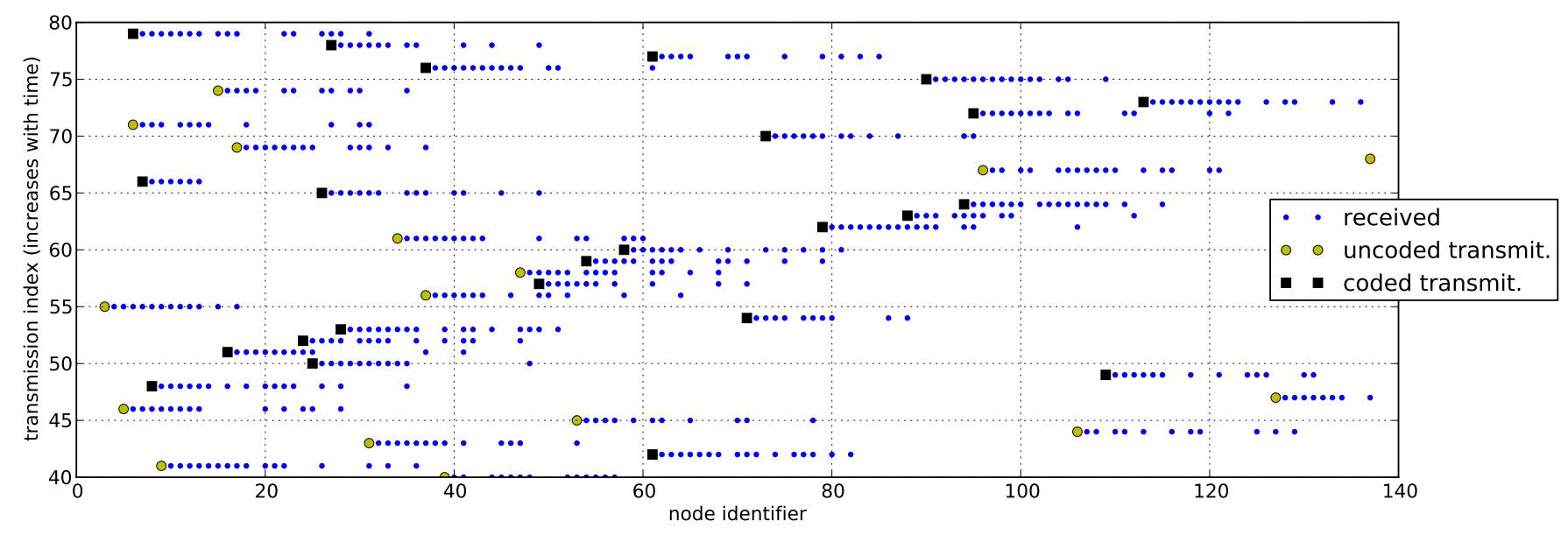

Figure 10. Sample diagram of received transmissions with a Rayleigh model

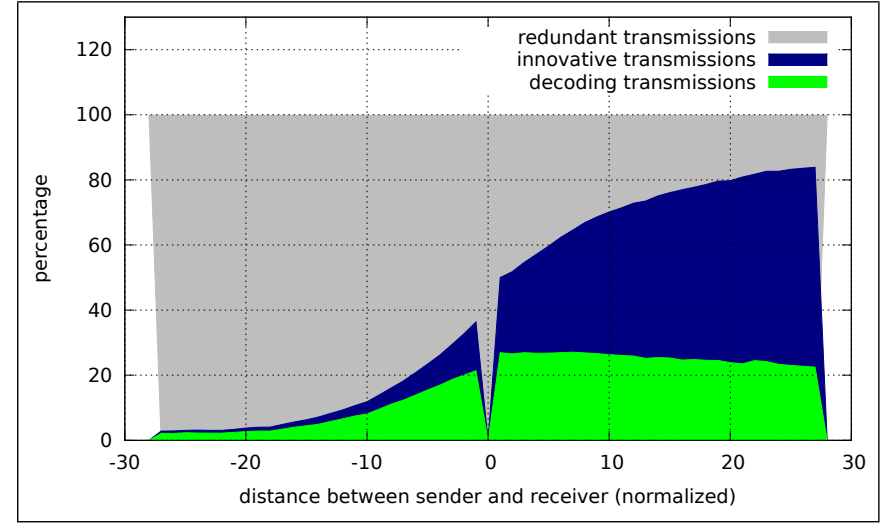

(a) transmissions with coded packets

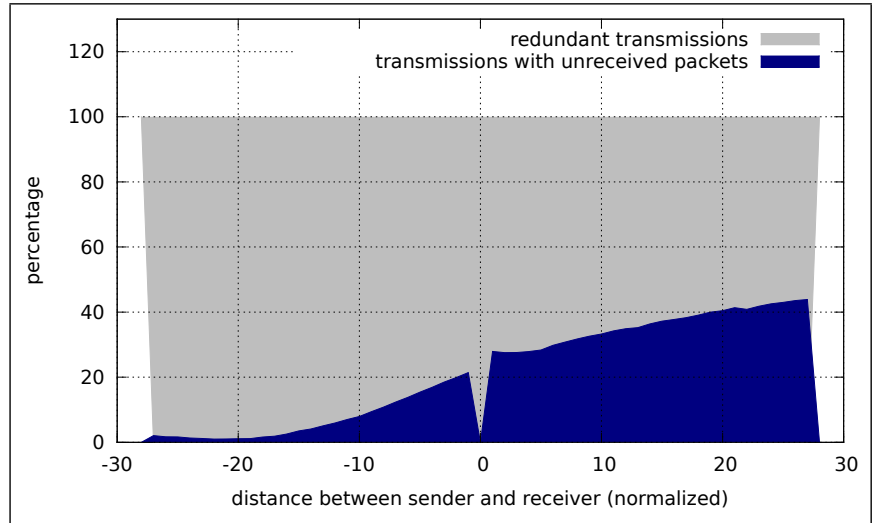

(b) transmission without coding

Figure 11. "Decoding", "innovative", and redundant transmissions' percentages, depending on distance between receiver and sender

coding (coding only a few packets), the performance is noticeably improved (as shown in next section), at the expense of a very small CPU cost overhead.

The last aspect in the opportunistic and "ad hoc" behavior of the protocol: it can be deduced that multiple packets are transmitted in parallel, but they are coded by some nodes, not coded by other nodes, with transmissions appearing at irregular times. The seemingly erratic transmission patterns actually originate from a strong point of the protocol because it reflects the fact that it does not require synchronized, predefined, rigid communication patterns. On the contrary, each node is acting on its own, with minimal information and feedback from neighboring nodes, and most importantly adapts to the current state of the network as it perceives it, including transmission losses. As a result, an efficient broadcast process emerges from the adaptive local behavior of each node.

In the figure 11, we represent statistics with DONC. It is a summary of the statistics of 50 simulations with the same parameters as for figure 10. For each transmission occurring in the simulation, we observed its effect on every receiver, depending also on whether the packet was coded or not, several cases are possible:

- with a coded packet (linear combination of 2 source packets or more), for the receiver, either:
- it is a "decoding" transmission, that is a transmission that allows the receiver to actually decode some packets

- or it is a "innovative" transmission, here defined as a transmission that does not allow to decode immediately, but that provides new information that would be decoded later

- or else it is a "redundant" transmission

- with a non-coded packet, for the receiver:

- the packet was not yet received

- or the packet was already received, and is redundant

Statistics of every occurring case were collected, depending on the distance between the receiver and the sender (normalized: 1 unit correspond to the distance between one vehicle and the one immediately behind it).

The figure 11 shows clearly the expected difference between upstream and downstream: downstream transmissions are noticeably more likely to be useful (non-redundant) than upstream transmissions; but we observe that some upstream transmissions are nonredundant, and are actually allowing packet loss recovery. Yet the difference between coding and not coding, is not dramatic for upstream transmissions. The largest difference appears with packets in the forward 
direction of the broadcast, where coded packets bring approximatively twice as much non-redundant information as non-coded packets. It is a perfect depiction of the improvements offered by the concept of network coding.

\subsection{Simulation Results}

In the simulations, almost all nodes (i.e. excluding a few downstream sources that do not receive other sources upstream) ultimately receive and decode the source packets. Then, the meaningful performance metric is the amount of redundancy, characterized by the ratio of average number of received packets per source packet (lower is better). In an ideal dense linear network without losses, the average number of receptions would be equal to 2: one reception from downstream and one reception from upstream (as it is further propagated by one repeater).

Figure 12(a) compares the performance of the DONC protocol with the SDB protocol in 2RM loss model. It clearly appears that for the vehicle densities ranging from 10 to $45 \mathrm{veh} / \mathrm{km}$, the average number of packet receptions per vehicle with the DONC protocol remains lower than with SDB. This is because the SDB protocol suffers increased packet redundancy to counter the effects of packet loss in VANETs, thereby increasing the network traffic as well as total channel occupancy of the network. On the other hand, the DONC protocol uses principles of network coding to reduce the number of redundant packet transmissions by encoding multiple messages in a coded packet. Lower unneeded packet redundancy with the DONC protocol translates into lower channel occupancy and lower wireless data traffic for the same amount of information to be communicated. Figure 12(a) illustrates that DONC protocol performance is very efficient in slightly lossy VANETs set for rural environments (2RM model). It keeps the mean number of receptions close to 2, thus almost reaching the optimal. Also, Figure 12(b) indicates that the number of receptions remains significantly inferior in highly congested and lossy environments. The Rayleigh loss model is adapted to complex urban centres where wireless medium quality is poor and wireless signals may be susceptible to heavy multipath fading. It can be seen in the figure 12(b) that the difference in performance of DONC over SDB is even greater than it was for 2RM model. This is because the Rayleigh loss model simulates much higher packet loss rates, and thus SDB increases its packet redundancy to cover for the increased packet loss. On the other hand, DONC encodes multiple messages in individual coded packets to reduce the packet redundancy and achieve better network performance.

Results presented in figures 12(a) and 12(b) show performance improvements brought by DONC over SDB in light as well as in heavily loss-riddled environments. While this is sufficient for real environments where losses occur mainly due to average/poor radio coverage, we should also make sure that DONC protocol performs equally, if not better than the SDB

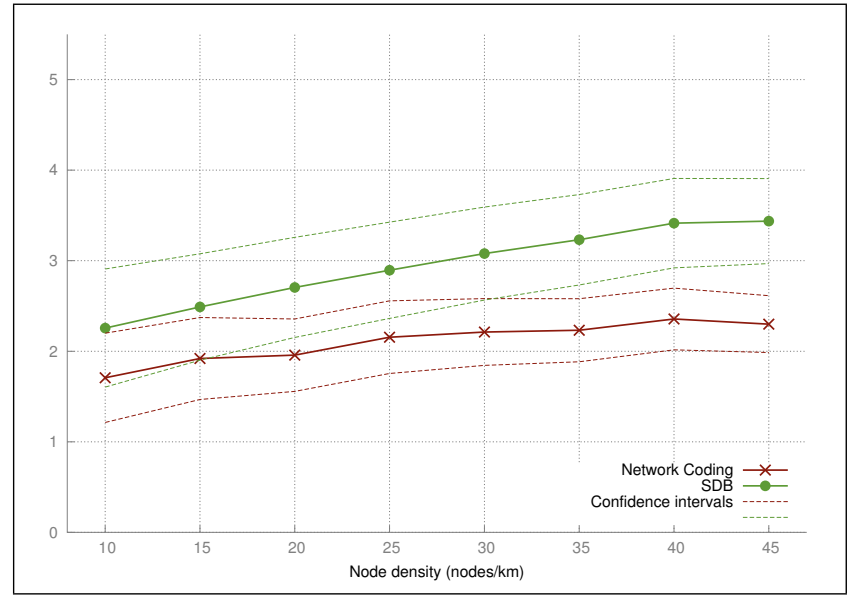

(a) $2 \mathrm{RM}$

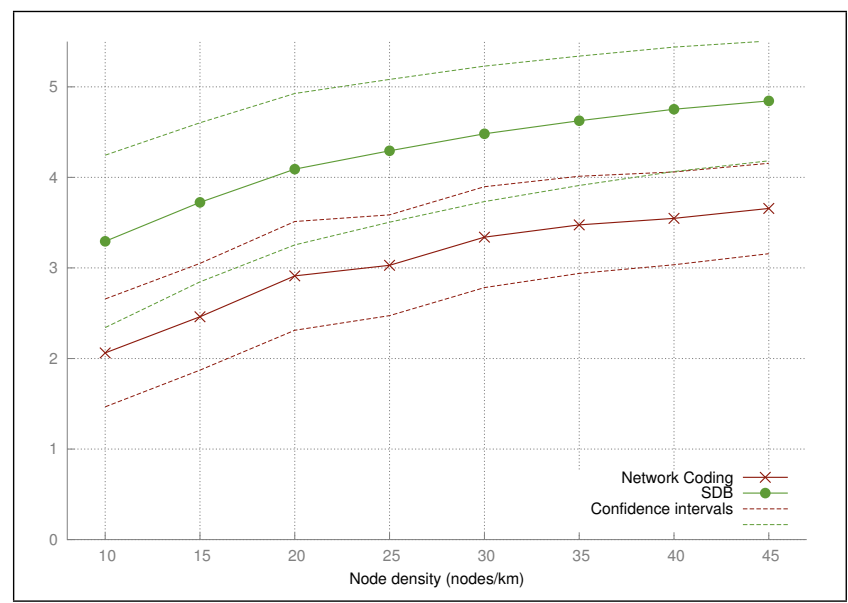

(b) Rayleigh

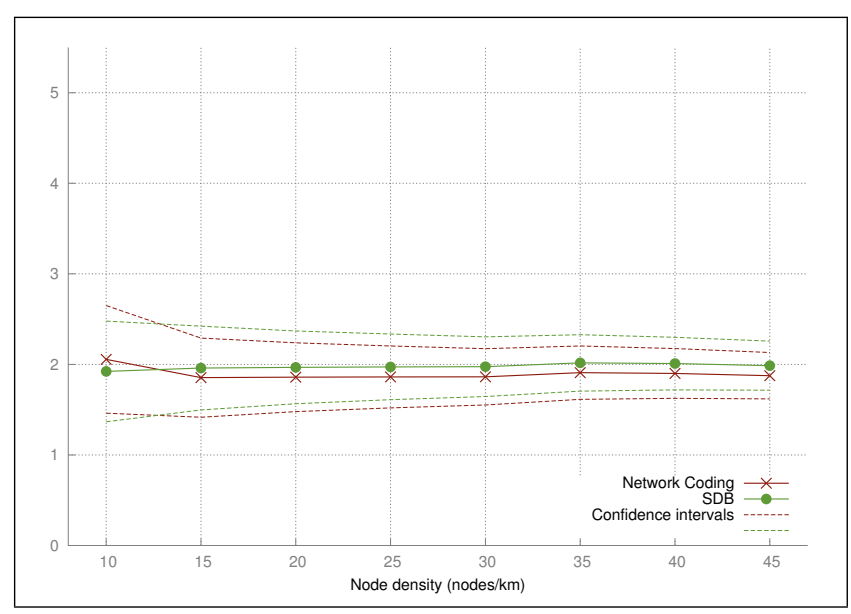

(c) Boolean

Figure 12. Average receptions per vehicle

protocol in an ideal environment with perfect radio reception, in order to check for any overhead. Figure 12(c) presents a comparison between DONC and SDB in an ideal environment (Boolean loss model), where the only losses possible are due to the collisions among different broadcasts. It can be seen in the figure that the performance of the DONC protocol in an ideal environment is as good as for the SDB protocol, both staying close to the ideal value of 2 .

Figure 13 further consolidates the results by showing DONC protocol's reduced number of transmissions per 


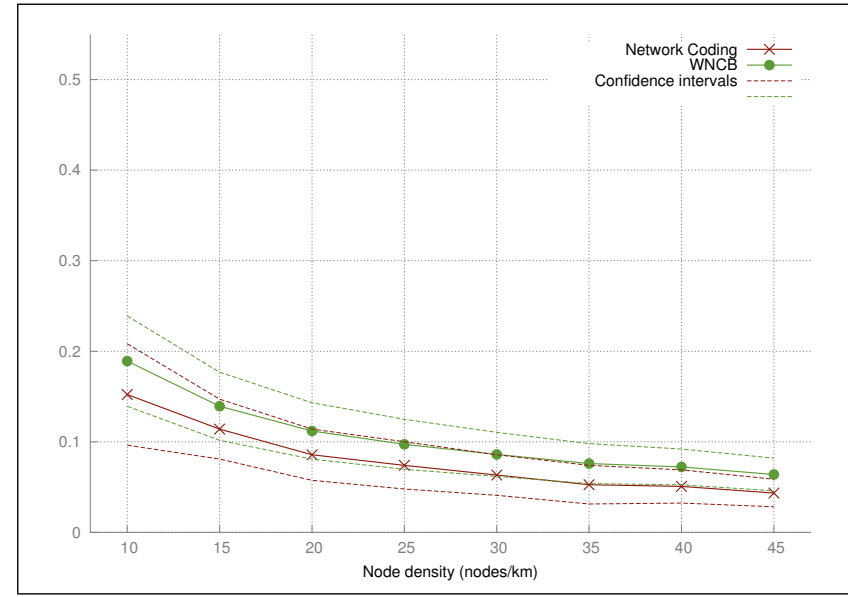

(a) $2 \mathrm{RM}$

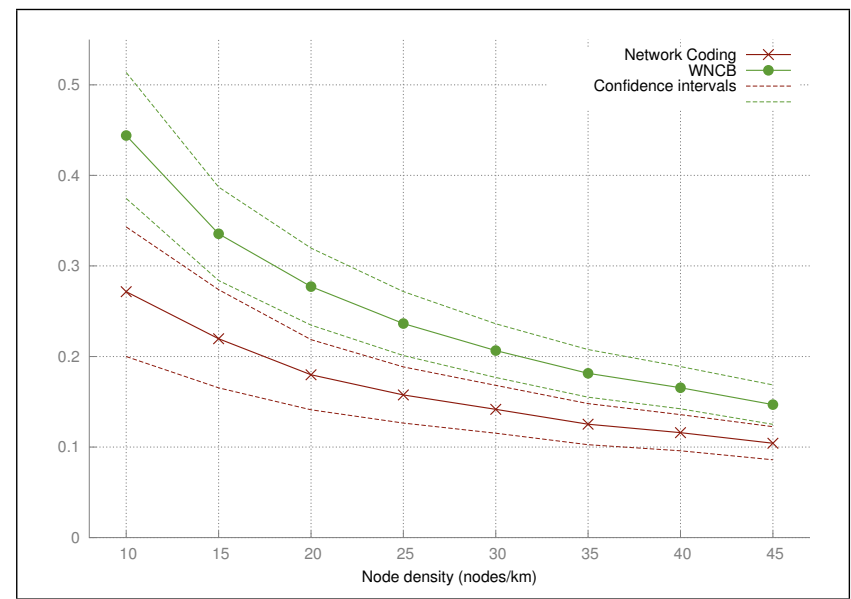

(b) Rayleigh

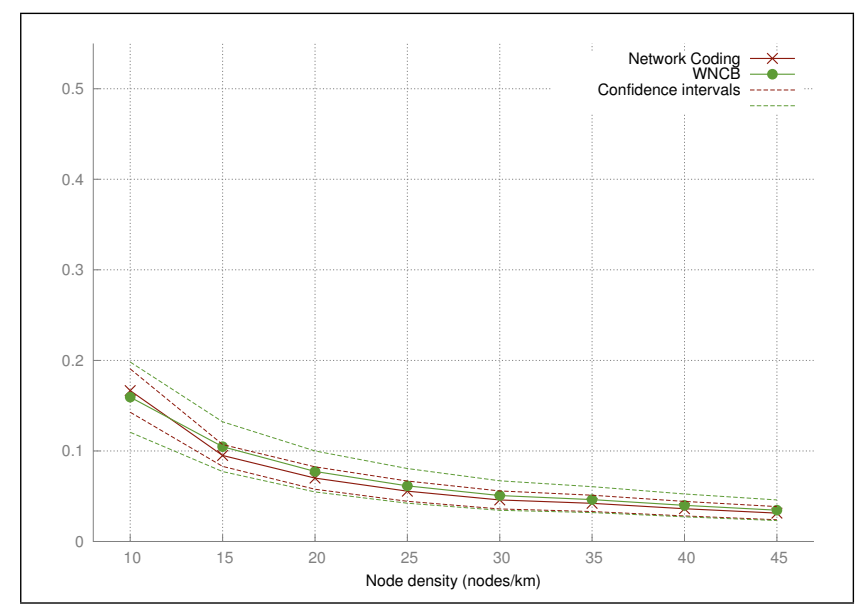

(c) Boolean

Figure 13. Average transmissions per vehicle

vehicle in comparison to SDB mechanism. It reduces the mean number of transmissions from $50 \%$ to $30 \%$ for the 2RM and Rayleigh models.

\section{Related Work}

This section is further divided into two subsections. First, we outline some important works on data dissemination and broadcast in VANETs and the second subsection briefly overviews network coding and some related works.

\subsection{VANET Relaying}

Packets travel through multi-hop broadcast networks by way of flooding. Ideally, a vehicular node (source) will transmit an information packet in broadcast mode and all the neighboring vehicles (receivers) in its transmission vicinity will receive the packet. Each of these receiving vehicles will act as relaying nodes and rebroadcast the received packet to their neighbors and so forth. In this way, the information packet may propagate through the vehicular network.

However, a vanilla flooding mechanism is most likely to be inefficient as every single vehicle that receives a packet will rebroadcast it, causing redundant transmissions wasting scarce radio resources. The problem is further aggravated as the network becomes denser, where all the receiving vehicles may broadcast at the same time, causing packet collisions. This is referred to as broadcast storm problem. There are several main techniques used to solve the above mentioned problems including: optimized flooding (with connected dominated sets), probabilistic broadcasting and delay-based broadcasting.

In the connected dominating set approach, a (precomputed) subset of the nodes retransmits the messages. In probabilistic broadcasting, different rebroadcast probabilities (also called forwarding probability) are assigned to each vehicle in the receiving range of a source vehicle, and again only a fraction of all the receiving vehicles rebroadcast a received packet, but this time, the set of such nodes is probabilisticly determined. Both approaches reduce the redundancy of a transmitted packets as well as packet collisions in a broadcast network. The biggest challenge for this type of rebroadcasting protocol to work efficiently is to determine the optimal probability for each vehicle. The simple techniques fix a probability value for each vehicle in a network, while the more sophisticated ones allow each vehicle to dynamically set its own probability value based on its current position with respect to its neighbors, network density, etc. An example is provided by the authors of [12] who propose a distancebased probabilistic broadcasting mechanism. Upon reception of a packet, the mechanism determines its forwarding probability based on the distance between the receiving and source vehicles. Farther the receiving vehicle, higher the probability of rebroadcast.

Just as probabilistic broadcasting is based on calculating an optimal value for forwarding probability, delay-based broadcasting mechanisms are based on determining the optimal delay on each receiving node before the packet can be rebroadcasted. Each receiving vehicle will wait for a calculated time period before it may rebroadcast the packet. The highest priority rebroadcasting vehicle will be the one with shortest waiting delay. Once a packet is rebroadcasted, other neighboring vehicles may abort their waiting processes, knowing this packet has already been rebroadcasted. Ideally, the delay assigned to each vehicle is a function of distance between the receiving and transmitting vehicles, such that farther the receiving vehicle is from 
the transmitter, shorter the waiting time.

Urban Multi-hop Broadcast (UMB) [13] proposes to divide part of the road that is under the transmission coverage of a transmitting vehicle into multiple segments and prioritize the vehicles by the position of their road segments in reference to the transmitting vehicle. When a packet is to be broadcasted, UMB transmitter first sends a request-to-broadcast (RTB) control message and waits for a clear-to-broadcast (CTB) message in response from one of the receiving vehicles. Upon reception of a $\mathrm{RTB}$, each receiving vehicle will start transmitting a blackburst signal, for a time that will be determined by its distance from the transmitting vehicle. The farthest vehicle to have received the RTB will transmit the longest blackburst, and therefore is chosen implicitly as the forwarding node. Authors in [14] present Efficient Directional Broadcast (EDB). Although similar in approach to UMB, EDB does not use RTB/CTB control messaging. EBD makes use of the directional antennas to distinguish the two directions of packet propagation (forward and backward) and has a unique waiting time calculating function. Multi-hop Vehicular Broadcast (MHVB) [15] is also a delay-based multi-hop broadcasting protocol. Every time a packet is received by a vehicle, a waiting time is computed based on its distance from the source vehicle. Again, the waiting time is shortest for the farthest vehicle from the source. This farthest vehicle will transmit the first and all the other nodes which are still waiting to rebroadcast the packet will cancel their timers and abort the packet rebroadcast.

\subsection{Network Coding}

Originally proposed by R. Ahlswede in [3], the idea of network coding breaks the long established assumption that an intermediate network node has to forward the exact same data it received. Instead, information at intermediate nodes is allowed to be processed, mixed together and re-constructed before it may be relayed forward, as opposed to just store-and-forward. Application of network coding not only results in significant improvement in network throughput and robustness but also helps achieve the network capacity.

Ahlswede's work was followed by the work of $\mathrm{Li}$ et al. [16], who proved that linear codes are sufficient to achieve maximum capacity bounds for the multicast traffic (e.g., a butterfly network). Koetter and Medard [17] presented a solution on how to find the coefficient of the linear encoding/decoding functions and $\mathrm{Ho}$ et al. [6] extended those results to random coding approach. In addition to that, Chou et al. [7] proposed integration of coefficients used in the linear combinations in the packet header itself to facilitate the decoding of a combination at the receiver without employing a centralized mechanism. Chou et al. also gave the notion of grouping packets into batches for coding together.

Following the aforementioned pioneering works, there has been extensive research analysing different solutions as well as its possible applications for network coding. Network coding has been proven very useful in context of broadcast wireless networks, which most commonly suffer from multi-user interference and lack of centralized coordination.

Authors Fragouli et al., in their work [18] presented distributed network coding algorithms which address issues such as forwarding factor, managing generations and impact of transmissions in order to achieve energy savings. Katti et al. proposed COPE [19]. Although it is a network coding based unicast routing protocol, COPE uses key properties of wireless communications, which also encompass communication in broadcast mode. The three basic techniques in COPE are based on: (a) snooping or overhearing all the packets being transmitted in the wireless broadcast medium that the node can overhear, (b) encoding packets in a combination with the guarantee that the combination will be decoded at the destination and (c) keep track of all the packets the neighbors have received so far. This is achieved with the reception reports, broadcasted by each node periodically.

The idea of COPE was adapted for VANETs in [20], which proposed two distinct queues for coding/decoding packets, each in a different direction of the traffic on a road and looking for opportunities to broadcast an XOR-ed combination which may contain packets useful to both directions simultaneously. Similarly, authors in [21] propose CODEB, a network coding based broadcast protocol. Also inspired by COPE, CODEB covers broadcasting scenarios for ad hoc wireless networks, in which opportunistic coding is performed not just for intended next-hop neighbor but for all the neighbors surrounding a node. Another relevant work in the area is presented in [22] which reduces the number of transmissions required to flood packets in an ad hoc wireless network. The principle difference between DifCode and CODEB is that CODEB required all of its receivers to instantaneously decode the incoming combinations while DifCode contains a buffer that temporarily stores the coded combinations until enough information has been collected to decode them.

Chachulsky et al. proposed MORE [4], which is a distributed multipath opportunistic routing (OR) mechanism that imbibes the theory of wireless broadcast and network coding. MORE proposes that multiple intermediate nodes between a source and a destination combine 'native packets' of data into linearly independent coded combinations (packets). These coded versions are then forwarded towards their common destination. Since these coded packets are linearly independent of each other, it suffices to deliver any ' $N$ ' combinations to the destination to successfully decode ' $N$ ' native packets of data. Results prove that MORE improves communication performance. Zhang et al. [5] present Optimized Multipath Network Coding (OMNC), which optimizes MORE to work in a distributed environment by assigning each node an encoding and broadcast rate in a decentralized manner. OMNC explores the broadcast nature of the wireless medium and its possible path diversity, and takes advantage of network 
coding to adapt to lossy environment by controlling the rate at which native packets may be encoded and/or broadcasted.

For further details on broadcast in VANETs, network coding and its working principles, readers may refer directly to $[1-3,6,7,16,17]$.

\section{Conclusion}

In this article, we have designed a method to improve delay-based broadcast protocols in environments where they significantly deviate from the optimum: environments with lossy communications. Such environments are commonly found in VANETs.

One of our main ideas is to combine the central mechanism of such protocols with the use of network coding, which offers desirable properties of reliability and loss recovery without the need for costly control protocol. This is materialized by the proposal of the DONC protocol, our Delay-based Opportunistic Network Coding protocol. The precise functioning of DONC was detailed, including essential features such as the coding strategy, and delay-based timeout choices, and some important practical points.

DONC was implemented for the simulator ns2 (and with different propagation loss models) and the performance of the protocol was studied on representative scenarios. Its performance was compared with classical delay-based broadcast. Results illustrate the fact that our protocol outperforms classical delay-based broadcast by successfully recovering losses in packets, and providing multi-packet implicit acknowledgments. In addition, we confirm that in the case of networks without loss or with less losses, the protocol overhead remains limited, making DONC applicable for all network conditions.

\section{REFERENCES}

[1] S. Panichpapiboon and W. Pattara-atikom, "A review of information dissemination protocols for vehicular ad hoc networks," IEEE Communications Surveys Tutorials, vol. 14, no. 3, pp. $784-798$, quarter 2012.

[2] A. Busson, "Performance evaluation of broadcast protocols in VANET: a point process approach," in Proceedings of the 6th ACM symposium on Performance evaluation of wireless ad hoc, sensor, and ubiquitous networks, ser. PE-WASUN '09. New York, NY, USA: ACM, 2009, pp. 108-115. [Online]. Available: http://doi.acm.org/10.1145/1641876.1641896

[3] R. Ahlswede, N. Cai, S.-Y. Li, and R. Yeung, "Network information flow," IEEE Transactions on Information Theory, vol. 46, no. 4, pp. $1204-1216$, jul 2000.

[4] S. Chachulski, M. Jennings, S. Katti, and D. Katabi, "MORE: A Network Coding Approach to Opportunistic Routing," Massachusetts Institute of Technology, Computer Science and Artificial Intelligence Laboratory, Massachusetts Institute of Technology, Tech. Rep., June 2006.

[5] X. Zhang and B. Li, "Optimized Multipath Network Coding in Lossy Wireless Networks," in Proceedings of the 2008 The 28th International Conference on Distributed Computing Systems, ser. ICDCS '08. Washington, DC, USA: IEEE Computer Society, 2008, pp. 243-250. [Online]. Available: http://dx.doi.org/10.1109/ICDCS.2008.45
[6] T. Ho, M. Médard, R. Koetter, D. R. Karger, M. Effros, J. Shi, and B. Leong, "A random linear network coding approach to multicast," IEEE Transactions on Information Theory, vol. 52, no. 10, pp. 4413-4430, 2006.

[7] P. A. Chou, Y. Wu, and K. Jain, "Practical network coding," 2003.

[8] L. Keller, M. Jafari Siavoshani, C. Fragouli, K. Argyraki, and S. Diggavi, "Identity Aware Sensor Networks," in Proceedings of the IEEE Conference on Computer Communications (INFOCOM), 2009.

[9] "The Network Simulator 2 (NS-2)." [Online]. Available: http://www.isi.edu/nsnam/ns

[10] "IEEE 802.11p: Wireless access in vehicular environments (wave)." [Online]. Available: http:/ / www.ieee802.org

[11] P. Barsocchi, G. Oligeri, and F. Potorti, "Frame error model in rural wi-fi networks," in Modeling and $\mathrm{Op}$ timization in Mobile, Ad Hoc and Wireless Networks and Workshops, 2007. WiOpt 2007. 5th International Symposium on, april 2007, pp. $1-6$.

[12] N. Wisitpongphan, O. K. Tonguz, J. S. Parikh, P. Mudalige, F. Bai, and V. Sadekar, "Broadcast storm mitigation techniques in vehicular ad hoc networks," Wireless Commun., vol. 14, no. 6, pp. 84-94, Dec. 2007. [Online]. Available: http://dx.doi.org/10.1109/MWC.2007.4407231

[13] G. Korkmaz, E. Ekici, F. Özgüner, and U. Özgüner, "Urban multi-hop broadcast protocol for intervehicle communication systems," in Proceedings of the 1st ACM international workshop on Vehicular ad hoc networks, ser. VANET '04. New York, NY, USA: ACM, 2004, pp. 76-85. [Online]. Available: http:/ /doi.acm.org/10.1145/1023875.1023887

[14] D. Li, H. Huang, X. Li, M. Li, and F. Tang, "A distancebased directional broadcast protocol for urban vehicular ad hoc network," in Wireless Communications, Networking and Mobile Computing, 2007. WiCom 2007. International Conference on, sept. 2007, pp. $1520-1523$.

[15] T. Osafune, L. Lin, and M. Lenardi, "Multi-hop vehicular broadcast (mhvb)," in ITS Telecommunications Proceedings, 2006 6th International Conference on, june 2006, pp. 757 760.

[16] S. yen Robert Li, S. Member, R. W. Yeung, and N. Cai, "Linear network coding," IEEE Transactions on Information Theory, vol. 49, pp. 371-381, 2003.

[17] R. Koetter, M. Médard, and S. Member, "An algebraic approach to network coding," IEEE/ACM Transactions on Networking, vol. 11, pp. 782-795, 2003.

[18] C. Fragouli, J. Widmer, and J.-Y. Le Boudec, “A network coding approach to energy efficient broadcasting: From theory to practice," in INFOCOM 2006. 25th IEEE International Conference on Computer Communications. Proceedings, April 2006, pp. $1-11$.

[19] S. Katti, H. Rahul, W. Hu, D. Katabi, M. Medard, and J. Crowcroft, "XORs in the air: Practical wireless network coding," IEEE/ACM Transactions on Networking, vol. 16, no. 3, pp. $497-510$, June 2008.

[20] L. Haojie, Z. Dongdong, and Y. Chen, "Local-directed network coding in vehicular ad-hoc networks," in 2010 International Symposium on Intelligent Signal Processing and Communication Systems (ISPACS), Dec 2010, pp. 1 -4.

[21] L. Li, R. Ramjee, M. Buddhikot, and S. Miller, "Network coding-based broadcast in mobile ad-hoc networks," in INFOCOM 2007. 26th IEEE International Conference on Computer Communications. IEEE, May 2007, pp. 1739 1747.

[22] N. Kadi and K. A. Agha, "MPR-based flooding with distributed fountain network coding," in Ad Hoc Networking Workshop (Med-Hoc-Net), 2010 The 9th IFIP Annual Mediterranean, June 2010, pp. $1-5$. 


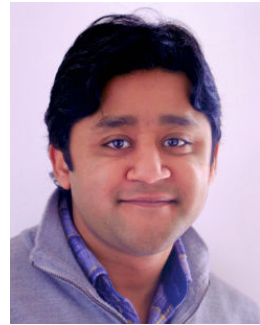

Farhan H. Mirani is currently a post-doctoral researcher with Laboratoire des Signaux et Systèms (LSS) at Ecole Supérieur d'Eléctricité (Supéléc), France. He had his B.E. degree from Mehran University of Engineering and Technology, Pakistan (2006), his Masters by Research degree from Université de Nice Sophia Antipolis, France (2008) and his PhD degree from Télécom ParisTech, France (2012). His primary research interests focus on heterogeneous access-network environments as well as vehicular ad-hoc networks. So far he has worked on scheduling algorithms, protocol designs, network coding, performance evaluation followed by data analysis, practical experimentation through testbeds. These experiments allowed him to achieve efficient multipath data transfers, path redundancy, bandwidth aggregation, dataloss masking, etc.

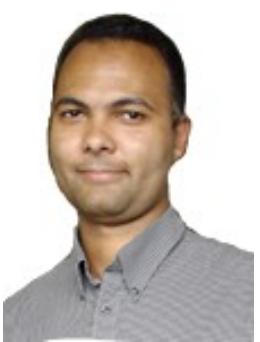

Cedric Adjih is currently a researcher in the Hipercom2 team at Inria. He received his $\mathrm{PhD}$ diploma in 2001 from Versailles University (France). He graduated from ECP (Ecole Centrale de Paris) in 1994. His research interests focus on wireless multihop networks (ad-hoc networks, mesh networks, sensor networks, VANETs). He is studying various aspects related to performance evaluation, algorithm and protocol design, and practical experimentation, on different subjects including: efficient routing, reliable routing, broadcasting and multicasting, ad-hoc military networks. energy-efficiency, quality of service, autoconfiguration, security, and also network coding. Cedric has worked on aspects of Mesh Networking within 802.11 task group "s" (with contributions to the 802.11s draft in 2005). He is co-author of the OLSR protocol (RFC 3626), and contributor to OLSRv2 (including RFC 5444).

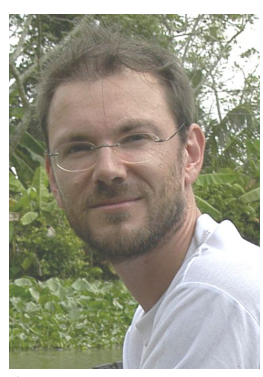

Anthony Busson is Professor at University Claude Bernard Lyon 1 (France) and at the Laboratoire de l'Informatique du Parallèlisme (Ecole Normale Supérieure de Lyon - INRIA University Lyon 1). He was associate professor at University Paris XI in 2003-2012, and at Laboratory of Signals and Systems (Supélec - CNRS - University Paris XI). He had his PhD from Télécom Paris Tech, France (2002), and his Master Degree in applied mathematics at University Paris VI. His research interests focus on protocol and performance evaluations of wireless networks. More specifically, some of these contributions deal with mobility, capacity, broadcast/dissemination in ad hoc and vehicular ad hoc networks. The performance evaluation tools are stochastic geometry, queuing theory, and more generally stochastic processes. 